\title{
On the $u$-invariant of a real function field
}

\author{
Karim Johannes Becher
}

\begin{abstract}
We obtain a new upper bound on the dimensions of anisotropic quadratic torsion forms over a field that is an extension of finite transcendence degree of a real closed field.
\end{abstract}

\section{Mathematics Subject Classification (2000) $\quad 11 \mathrm{E} 04 \cdot 11 \mathrm{E} 81 \cdot 12 \mathrm{D} 15$}

Let $K$ be a field of characteristic different from 2. We refer to [5] and [9] for the basic concepts of the theory of quadratic forms over fields. By a form we always mean a regular quadratic form. Let $W K$ denote the Witt ring of $K$ and $I K$ its fundamental ideal. For $n \in \mathbb{N}$ we set $I^{n} K=(I K)^{n}$ and write $I_{t}^{n} K$ for the torsion part of $I^{n} K$. We say that a quadratic form is torsion if it represents a torsion element in $W K$.

The $u$-invariant of $K$ was defined in [1] as

$$
u(K)=\sup \{\operatorname{dim}(\varphi) \mid \varphi \text { anisotropic torsion form over } K\} \in \mathbb{N} \cup\{\infty\}
$$

For nonreal fields this invariant has already been considered in [4]. Recall that $K$ is nonreal if -1 is a sum of squares in $K$, real otherwise. If $K$ is nonreal, then every quadratic form over $K$ is torsion and $u(K)$ is the smallest integer $n$ such that every quadratic form over $K$ of dimension $n$ is universal (i.e., it represents every element of $K$ ) if such an integer exists, otherwise $u(K)=\infty$. (This explains the choice of the letter $u$.)

We refer to [7, Chap. 8] for a survey on the $u$-invariant. One open problem is whether finiteness of the $u$-invariant is conserved under field extensions of finite transcendence 
degree. For example, it is not known whether $u(\mathbb{Q}(X))$ is finite. Recently, in [8] it was proven that $u(K)=8$ when $K$ is a function ficld of a $p$-adic curve for a prime $p \neq 2$. For a field extension $K / C$ of transcendence degree $n$ of an algebraically closed field $C$, Tsen-Lang Theory yields that $u(K) \leq 2^{n}$. For extensions $K / R$ of transcendence degree $n>2$ of a real closed field $R$, it was shown in $\left[2\right.$, Sect. 6] that $u(K)<2^{n+2}$.

The aim of this article is to prove the following statement:

$$
\text { If } n \geq 2 \text { is such that } u(K(\sqrt{-1})) \leq 2^{n} \text {, then } u(K) \leq 2^{n+2}-2 n-6 \text {. }
$$

It applies in particular to the case where $K$ is an extension of transcendence degree $n$ of a real closed field and, for $n \geq 3$, it improves the bounds known so far for this case. For $n=2$, one retrieves the observation from [1, Theorem 4.11] that $u(K(\sqrt{-1})) \leq 4$ implies $u(K) \leq 6$.

To obtain the result we will refine a method introduced in [1, Sect. 4] and sometimes referred to as the Elman-Lam filtration of the u-invariant. For a quadratic form $\varphi$ over $K$ and $n \in \mathbb{N}$, let $n \times \varphi$ denote the $n$-fold $\operatorname{sum} \varphi \perp \ldots \perp \varphi$. For $i \in \mathbb{N}$, let $\mathcal{S}_{i}(K)$ denote the set of anisotropic forms $\varphi$ over $K$ such that $2^{i} \times \varphi$ is hyperbolic, and put

$$
u^{i}(K)=\sup \left\{\operatorname{dim}(\varphi) \mid \varphi \in \mathcal{S}_{i}(K)\right\} \in \mathbb{N} \cup\{\infty\}
$$

We thus have an increasing sequence $\left(\mathcal{S}_{i}(K)\right)_{i \geq 0}$ of subsets of the set of all anisotropic forms over $K$ and an increasing sequence $\left(u^{i}(K)\right)_{i \geq 0}$ in $\mathbb{N} \cup\{\infty\}$. The set $\mathcal{S}_{0}(K)$ contains only the trivial quadratic form, so we have $u^{0}(K)=0$. Moreover, since every torsion form is annihilated by some power of 2 , the set $\cup_{i \geq 0} \mathcal{S}_{i}(K)$ consists of all anisotropic torsion forms over $K$, and therefore we have $u(K)=\sup _{i \geq 0} u^{i}(K)$. In [3], for any $k \geq 0$, fields $K$ were constructed with $u^{k+1}(K)>u^{k}(K)$.

The interest of this filtration lies in the possibility of relating the values $u^{i}(K)$ $(i \geq 1)$ to each other and comparing $u^{1}(K)$ with $u(K(\sqrt{-1})$ ). An argument from $[1$, Sect. 2] shows that any quadratic form has an orthogonal decomposition $\sigma \perp \tau$ such that $2 \times \sigma$ is anisotropic and $2 \times \tau$ is hyperbolic. Applying this for $i>1$ to the forms in $S_{i}(K)$ readily yields the inequality $u^{i}(K) \leq u^{1}(K)+\frac{1}{2} u^{i-1}(K)$. Hence, $u^{n}(K) \leq 2 u^{1}(K)$ holds for all $n \geq 1$, and therefore $u(K) \leq 2 u^{1}(K)$.

In [2, Theorem 6.2] it was shown that $u(K)<4 u(K(\sqrt{-1}))$ whenever $u(K(\sqrt{-1}))$ is finite. The proof in $[2$, p. 38$]$ is quickly rearranged to obtain the following.

Theorem 1 (Elman-Lam) Assume that -1 is not a square in $K$. Then $u^{1}(K) \leq$ $2(u(K(\sqrt{-1}))-1)$ and $u(K) \leq 4(u(K(\sqrt{-1}))-1)$.

Proof Let $s_{*}$ denote the quadratic form transfer for the quadratic extension $K(\sqrt{-1}) / K$ given by the $K$-linear map $s: K(\sqrt{-1}) \rightarrow K$ with $s(1)=0$ and $s(\sqrt{-1})=1$ (cf. [5, Chap. VII, Sect. 1]). Given $\varphi \in \mathcal{S}_{1}(K)$, it follows from [5, Chap. VII, Theorem 3.4] that $\varphi=s_{*}(\gamma)$ for a form $\gamma$ over $K(\sqrt{-1})$, and as $\varphi$ is anisotropic, $\gamma$ does not represent 1 over $K(\sqrt{-1})$. Hence, $\gamma$ is not universal over $K(\sqrt{-1})$ and therefore $\frac{1}{2} \operatorname{dim}(\varphi)=\operatorname{dim}(\gamma) \leq u(K(\sqrt{-1}))-1$. This proves the first inequality. As $u(K) \leq 2 u^{1}(K)$, the second inequality follows from the first. 
In order to improve the estimate $u(K) \leq 2 u^{1}(K)$ in certain cases, we introduce a second sequence of field invariants. For any positive integer $i$ we define $S_{i}^{\prime}(K)=$ $\left\{\varphi \in \mathcal{S}_{i}(K) \mid 2^{i-1} \times \varphi\right.$ anisotropic $\}$ and

$$
w^{i}(K)=\sup \left\{\operatorname{dim}(\varphi) \mid \varphi \in \mathcal{S}_{i}^{\prime}(K)\right\} \in \mathbb{N} \cup\{\infty\} .
$$

Obviously, $w^{i}(K) \leq u^{i}(K)$ holds for any $i \geq 1$, and $\mathcal{S}_{1}(K)=\mathcal{S}_{1}^{\prime}(K)$ yields that $w^{1}(K)=u^{1}(K)$. For any $i>1$, if $\varphi \in \mathcal{S}_{i}^{\prime}(K)$, then $2 \times \varphi \in \mathcal{S}_{i-1}^{\prime}(K)$, which shows the inequality $w^{i}(K) \leq \frac{1}{2} w^{i-1}(K)$. Thus $\left(w^{i}(K)\right)_{i \geq 1}$ is a decreasing sequence in $\mathbb{N} \cup\{\infty\}$.

When saying that an element of $\mathbb{N} \cup\{\infty\}$ is even, we allow it to be $\infty$. Recall that the level of $K$, denoted by $s(K)$, is the (additive) order of the form $\langle 1,1\rangle$ in the Witt ring $W K$ (cf. [5, Chap. XI, Sect. 2]).

Proposition 1 Let $n \geq 1$. If $s(K) \geq 2^{n}$, then $u^{n}(K)$ and $w^{n}(K)$ are even. If $s(K)<$ $2^{n}$, then $u(K)=u^{n}(K)$ and $w^{i}(K)=0$ for $i>n$.

Proof Assume that $s(K) \geq 2^{n}$. Then $2^{n} \times\langle 1\rangle$ is not hyperbolic. Since every zero divisor in $W K$ is contained in $I K$ (cf. [5, Chap. VIII, Corollary 8.5]), it follows that $\mathcal{S}_{n}(K)$ contains only forms of even dimension. In particular, $u^{n}(K)$ and $u^{n}(K)$ are even. Assume now that $s(K)<2^{n}$. Then $2^{n} \times\langle 1\rangle$ is hyperbolic, so $\mathcal{S}_{n}(K)$ consists of all anisotropic forms over $K$ and $\mathcal{S}_{i}^{\prime}(K)$ contains only the zero form for $i>n$. Hence, $u(K)=u^{n}(K)$ and $w^{i}(K)=0$ for $i>n$.

Lemma Let $n \geq 0$. For every form $\varphi$ over $K$ there is a form $\psi$ over $K$ such that $2^{n} \times \psi$ is the anisotropic part of $2^{n} \times \varphi$.

Proof This follows from [5, Chap. X, Theorem 4.11] applied to the Pfister form $2^{n} \times\langle 1\rangle$ and the anisotropic part of $2^{n} \times \varphi$.

Proposition 2 If $n>1$, then $u^{n}(K) \leq u^{n-1}(K)+w^{n}(K)$.

Proof Let $n>1$. Let $\varphi \in S_{n}(K)$. It follows from the Lemma that the anisotropic part of $2^{n-1} \times \varphi$ is equal to $2^{n-1} \times \gamma$ for a form $\gamma$ over $K$. Then $\gamma \in \mathcal{S}_{n}^{\prime}(K)$, so $\operatorname{dim}(\gamma) \leq w^{n}(K)$. Let $\psi$ be the anisotropic part of $\varphi \perp-\gamma$. Then $2^{n-1} \times \psi$ is hyperbolic, so $\psi \in \mathcal{S}_{n-1}(K)$ and $\operatorname{dim}(\psi) \leq u^{n-1}(K)$. Since $\varphi$ is anisotropic and Witt equivalent to $\psi \perp \gamma$, we have $\operatorname{dim}(\varphi) \leq \operatorname{dim}(\psi)+\operatorname{dim}(\gamma) \leq u^{n-1}(K)+w^{n}(K)$. This shows that $u^{n}(K) \leq u^{n-1}(K)+w^{n}(K)$.

Corollary For any $n \geq 1$, we have $u^{n}(K) \leq \sum_{i=1}^{n} w^{i}(K)$.

Proof Since $u^{1}(K)=w^{1}(K)$, this follows from Proposition 2.

Theorem 2 Let $n \geq 2$ be such that $I_{t}^{n+1} K=0$. Then

$$
u(K) \leq u^{n-2}(K)+w^{n-1}(K) \leq \sum_{i=1}^{n-1} w^{i}(K) .
$$

Moreover, $w^{n-1}(K)$ is either even or equal to 1. 
Proof Suppose that $\rho$ is a form over $K$ such that $2^{n-2} \times \rho$ is anisotropic and represents an element of $I_{t}^{n} K$. Then for any form $\gamma$ over $K$ with $\operatorname{dim}(\gamma)=2$, it follows that $2^{n-2} \times \rho \otimes \gamma$ represents an element of $I_{t}^{n+1} K=0$ and therefore is hyperbolic. This shows in particular that $\rho \in \mathcal{S}_{n-1}^{\prime}(K)$ and thus $\operatorname{dim}(\rho) \leq w^{n-1}(K)$, and that either $2^{n-2} \times \rho$ is universal or the zero form. Moreover, since all zero divisors in $W K$ are contained in $I K$, it follows that $\rho$ has even dimension.

Suppose now that $\psi$ is a torsion form over $K$ with $\operatorname{dim}(\psi)>2$ such that $2^{n-2} \times \psi$ is anisotropic. Let $d$ denote the discriminant (signed determinant) of $\psi$. Set $\beta=\langle 1,-d\rangle$ if $\psi$ has even dimension and $\beta=\langle d\rangle$ otherwise. Then $\psi \perp-\beta$ represents an element of $I_{t}^{2} K$ and $2^{n-2} \times(\psi \perp-\beta)$ is not hyperbolic. By the Lemma, there is a form $\rho$ over $K$ such that $2^{n-2} \times \rho$ is the anisotropic part of $2^{n-2} \times(\psi \perp-\beta)$. Then $2^{n-2} \times \rho$ represents a nonzero element of $I_{t}^{n} K$. Hence, by the above $\operatorname{argument}, \operatorname{dim}(\rho)$ is even, $\operatorname{dim}(\rho) \leq w^{n-1}(K)$, and $2^{n-2} \times \rho$ is universal. In particular, $2^{n-2} \times(\rho \perp \beta)$ is isotropic. As $2^{n-2} \times \psi$ is the anisotropic part of $2^{n-2} \times(\rho \perp \beta)$, we obtain that $\operatorname{dim}(\psi)<\operatorname{dim}(\rho)+\operatorname{dim}(\beta)$. Therefore $\operatorname{dim}(\psi) \leq \operatorname{dim}(\rho) \leq w^{n-1}(K)$. Using this for all $\psi \in \mathcal{S}_{n-1}^{\prime}(K)$ shows that $w^{n-1}(K)$ is even or equal to 1 .

If $w^{n-1}(K)<2$, then for $i \geq n$ we have $w^{i}(K)=0$ and $u^{i}(K)=u^{i-1}(K)$ by Proposition 2, thus $u(K)=u^{n-1}(K) \leq u^{n-2}(K)+w^{n-1}(K)$. Assume now that $w^{n-1}(K) \geq 2$. Let $\varphi$ be an arbitrary anisotropic torsion form over $K$. By the Lemma, there exists a form $\psi$ over $K$ such that $2^{n-2} \times \psi$ is the anisotropic part of $2^{n-2} \times \varphi$. Then $\psi$ is torsion and the above arguments show that $\operatorname{dim}(\psi) \leq w^{n-1}(K)$. Since the anisotropic part of $\varphi \perp-\psi$ is an element of $\mathcal{S}_{n-2}(K)$ and since $\varphi$ is the anisotropic part of $(\varphi \perp-\psi) \perp \psi$, it follows that $\operatorname{dim}(\varphi) \leq u^{n-2}(K)+w^{n-1}(K)$. This shows the first inequality in the statement. The second inequality is clear from the Corollary.

Theorem 3 Let $n \geq 2$ be such that $u(K(\sqrt{-1})) \leq 2^{n}$. Then

$$
u(K) \leq 2^{n+2}-2 n-6
$$

Proof The hypothesis implies that $I_{t}^{n+1} K=0$ (cf. [5, Chap. XI, (6.27)]). By Theorem 1 , we have $w^{1}(K)=u^{1}(K)<2 u(K(\sqrt{-1})) \leq 2^{n+1}$. Recall that $w^{i}(K) \leq$ $\frac{1}{2} w^{i-1}(K)$ for $i>1$. We thus have $w^{i}(K)<2^{n-i+2}$ for all $i \geq 1$. If $w^{i}(K)$ is even for every $i \leq n-1$, then using Theorem 2 we conclude that $u(K) \leq \sum_{i=1}^{n-1} w^{i}(K) \leq$ $\sum_{i=1}^{n-1}\left(2^{n-i+2}-2\right)=2^{n+2}-2 n-6$. Assume now that $w^{m}(K)$ is odd for some $m \leq n-1$. By Proposition 1 , then $s(K)=2^{m-1}, u(K)=u^{m}(K)$, and, for $1 \leq$ $i<m, w^{i}(K)$ is even and thus $w^{i}(K) \leq 2^{n-i+2}-2$. Moreover, if $m=n-1$, then $u^{m}(K)=1$, by Theorem 2 . Using the Corollary, we now obtain the chain of inequalities $u(K)=u^{m}(K) \leq \sum_{i=1}^{m} w^{i}(K) \leq 1+\sum_{i=1}^{n-2}\left(2^{n-i+2}-2\right) \leq 2^{n+1}-2 n-3$. As $n \geq 2$, we have $2^{n+1} \geq 8$ and thus even $u(K) \leq 2^{n+2}-2 n-11$.

It follows from [6] that there exists a field $K$ such that $u(K)=6$ and $u(K(\sqrt{-1}))=$ 4. For $n=3$ Theorem 3 states that $u(K(\sqrt{-1})) \leq 8$ implies $u(K) \leq 20$. It is therefore interesting to ask whether a field $K$ with $u(K)=20$ and $u(K(\sqrt{-1}))=8$ does exist.

Acknowledgments This work was supported by the Deutsche Forschungsgemeinschaft (project Quadratic Forms and Invariants, BE 2614/3-1) and by the Zukunftskolleg, Universität Konstanz. 


\section{References}

1. Elman, R., Lam, T.Y.: Quadratic forms and the $u$-invariant, 1 . Math. Z. 131, 283-304 (1973)

2. Elman, R., Lam, T.Y.: Quadratic forms under algebraic extensions. Math. Ann. 219, 21-42 (1976)

3. Hoffmann, D.W.: On Elman and Lam's filtration of the $u$-invariant. J. Reine Angew. Math. 495, 175186 (1998)

4. Kaplansky, I.: Quadratic forms. J. Math. Soc. Jpn. 5, 200-207 (1953)

5. Lam, T.Y.: Introduction to quadratic forms over fields. Graduate Studies in Mathematics, vol . 67. American Mathematical Society, Providence (2005)

6. Mináč, J., Wadsworth, A.R.: The $u$-invariant for algebraic extensions. $K$-theory and algebraic geometry: connections with quadratic forms and division algebras-Santa Barbara, 1992. Proc. Sympos. Pure Math., pp. 333-358, vol. 58. Amer. Math. Soc., Providence (1995)

7. Pfister, A.: Quadratic forms with applications to algebraic geometry and topology. London Mathematical Society Lecture Notes series, vol. 217. Cambridge University Press, Cambridge (1995)

8. Parimala, R., Suresh, V.: The $u$-invariant of the function fields of $p$-adic curves (preprint, 2007). Online (arXiv): http://front.math.ucdavis.edu/0708.3128

9. Scharlau, W: Quadratic and Hermitian forms. Grundlehren der Mathematischen Wissenschaften, vol. 270. Springer, Berlin (1985) 